\title{
TITAN: Erlotinib vergleichbar wirksam wie Chemotherapie
}

\begin{abstract}
Bei Patienten mit fortgeschrittenem nichtkleinzelligem Lungenkarzinom (NSCLC) und ungünstiger Prognose ist die Second-line-Therapie mit dem oralen EGFR-Inhibitor Erlotinib ebenso wirksam wie eine Standardchemotherapie, verursacht aber weniger schwerwiegende Nebenwirkungen. Dies zeigen die Ergebnisse der randomisierten offenen Phase-III-Studie TITAN.
\end{abstract}

Patienten mit Progression unter einer First-line-Chemotherapie haben eine sehr ungünstige Prognose. In der Studie TITAN (Tarceva In Treatment of Advanced NSCLC) wurde in diesem Patientenkollektiv erstmals Erlotinib direkt mit einer Standardchemotherapie (Docetaxel bzw. Pemetrexed nach Entscheidung des Studienarztes) verglichen. Primärer Endpunkt war das Gesamtüberleben (OS). Zu den sekundären Endpunkten zählten neben dem progressionsfreien Überleben und der Ansprechrate auch die Sicherheit sowie Biomarker-Analysen.

\section{Keine signifikanten Unterschiede im Gesamtüberleben ...}

Bei einem medianen OS von 5,3 Monaten im Erlotinib-Arm ( $n=203)$ und 5,5 Monaten im Vergleichsarm $(n=221)$ war der Unterschied im Sterberisiko nicht signifikant ( $p=0,7299 ; A$ Abb.). Die Gleichwertigkeit beim primären Endpunkt wurde auch in allen Subgruppen und somit unabhängig von prädiktiv und prognostisch relevanten Parametern, wie z.B. Geschlecht, Raucherstatus und Tumorhistologie sowie Krankheitsstadium, Allgemeinzustand und ethnischem Hintergrund, gezeigt. Patienten mit nicht mutiertem EGFR (Epidermal Growth Factor Receptor) profitierten ebenso von Erlotinib wie das Gesamtkollektiv (OS Erlotinib versus Chemotherapie: Hazard-Ratio 0,85; 95-\%-Konfidenzintervall 0,59-1,22).

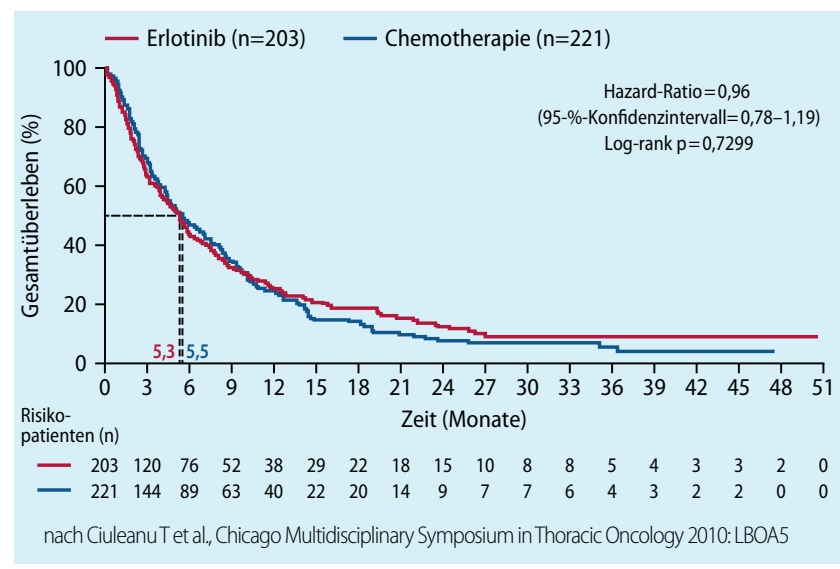

Primärer Endpunkt der TITAN-Studie: Gesamtüberleben

... unter Erlotinib aber weniger schwerwiegende Nebenwirkungen Die Therapie mit Erlotinib war mit weniger schwerwiegenden Nebenwirkungen assoziiert als die Standardchemotherapie. Hämatologische Toxizitäten traten unter Erlotinib nicht auf. Als häufigste Nebenwirkungen manifestierten sich im Erlotinib-Arm überwiegend leichte bis moderate Fälle von Rash und Diarrhö.

\section{Fazit}

Erstmals konnte im direkten Vergleich gezeigt werden, dass secondline Erlotinib ebenso wirksam ist wie eine Standardchemotherapie, die allerdings mehr schwerwiegende Nebenwirkungen verursacht.

Quellen: Ciuleanu T et al., Chicago Multidisciplinary Symposium in Thoracic Oncology 2010: LBOA5; Informationen der Roche Pharma AG

\section{Supportivtherapie \\ Informationsportal zu Biosimilars im Internet}

Umfassendes, verständliches und von Experten bestätigtes Wissen über Biosimilars das zeichnet die Website www.BiosimilarsInfo.de aus. Als eine der ersten deutschsprachigen Websites zu diesem Thema informiert sie detailliert über die Nachfolgepräparate biopharmazeutischer Arzneimittel, deren Patentschutz abgelaufen ist.

Auf www.Biosimilars-Info.de finden Interessierte Antworten auf Fragen rund um Biosimilars. Das Portal bündelt bereits vorhandene Informationen und macht sie mit wenigen Klicks verfügbar. Es ist in Koope- ration mit einem interdisziplinären, unabhängigen, wissenschaftlichen Beirat namhafter Experten entstanden, der sämtliche Inhalte diskutiert und autorisiert hat. Im Mittelpunkt des Wissensangebots stehen der Herstellungsprozess, das Zulassungsverfahren, das für Wirksamkeit, Qualität und Unbedenklichkeit bürgt, sowie die Unterschiede zwischen Biosimilars und herkömmlichen Generika. Außerdem wird das Einsparpotenzial verdeutlicht, das durch den Einsatz von Biosimilars erzielt werden kann. Die Rubrik „Aktuelles“ bietet Informationen über neue Entwicklungen auf dem Gebiet der Biosimilars. Unter "Literatur \& Links" ist eine aktuelle Übersicht zu Veröffentlichungen zu finden.

Europaweit sind mittlerweile bereits 15 Biosimilars zur Behandlung bei Krebs, chronischer Nierenerkrankung oder Wachstumsstörungen verfügbar. Sowohl die Präparateanzahl als auch die Zahl der Einsatzgebiete wird Experten zufolge steigen. Damit steigt auch der Informationsbedarf. HEXAL möchte als Initiator von www.Biosimilars-Info.de das Informationsangebot verbessern und Transparenz schaffen.

Quelle: Informationen der HEXAL AG 